Review Article

\title{
Endophthalmitis
}

\author{
Shwu-Jiuan Sheu ${ }^{1,2}$ \\ ${ }^{1}$ Department of Ophthalmology, Kaohsiung Veterans General Hospital, Kaohsiung, Taiwan \\ ${ }^{2}$ School of Medicine, National Yang-Ming University, Taipei, Taiwan
}

\begin{abstract}
Endophthalmitis is a rare but severe form of ocular inflammation due to infection of the intraocular cavity that can lead to irreversible visual loss if not treated properly and timely. It can be classified as exogenous or endogenous based on the transmission route of the infectious source. Exogenous endophthalmitis occurs when infecting organisms gain entry into the eye via direct inoculation, while endogenous endophthalmitis occurs when infectious agents hematogenously spread into the eye from a distant focus of infection. The diagnosis of endophthalmitis depends mostly on the clinical findings on ophthalmological examination. Delayed diagnosis of endogenous endophthalmitis can lead to not only visual loss, but also increased risk of mortality. Since ocular and systemic symptoms of endophthalmitis are usually non-specific, early diagnosis relies on the alertness of clinicians. Early diagnosis and proper treatment are keys to saving the eye. Following advances in vitreoretinal pharmacotherapy and surgical technology, early surgical intervention is the current trend in the management of endophthalmitis.
\end{abstract}

Key Words: Endogenous, Endophthalmitis, Exogenous

Endophthalmitis is a rare but severe form of ocular inflammation due to infection of the intraocular cavity that can lead to irreversible visual loss if not treated properly and timely. Based on the transmission route of the infection, endophthalmitis can be classified as exogenous or endogenous. Exogenous endophthalmitis occurs when infecting organisms gain entry into the eye via direct inoculation, such as from intraocular surgery, penetrating trauma, or contiguous spread from adjacent tissues. Endogenous endophthalmitis occurs when infectious agents are hematogenously spread into the eye from a distant focus of infec-

Received: March 14, 2017 Accepted: March 17, 2017

Corresponding Author: Shwu-Jiuan Sheu, MD, PhD. Department of Ophthalmology, Kaohsiung Veterans General Hospital, \#386 Ta-Chung 1st Rd, Kaohsiung 813, Taiwan. Tel: 886-7-346-8217, Fax: 886-7-346-8054, E-mail: sjiuansheu@gmail.com tion [1]. Endogenous endophthalmitis is generally less common than exogenous endophthalmitis, although the proportion of endogenous endophthalmitis ranged widely from $2 \%$ to $41 \%$ in different reports due to geographic, genetic, or alimentary factors [2-4]. In a British review of acute endophthalmitis, Krause et al. [4] reported that up to $41 \%$ of cases were endogenous. However, only $7.4 \%$ of 955 endophthalmitis cases were endogenous in a report from India [5]. In our review of 326 cases of endophthalmitis from 2002 to 2013 in a tertiary medical center in Taiwan, endogenous endophthalmitis accounted for $26.4 \%$ of all cases (unpublished data).

\section{Endogenous Endophthalmitis}

In endogenous endophthalmitis, gram-positive bacteria, 
gram-negative bacteria, and fungus are among the frequently reported pathogens. In general, gram-negative organisms, especially Klebsiella pneumoniae (KP), are responsible for most cases of endogenous endophthalmitis in East Asia, whereas gram-positive organisms and fungi are the more frequent causative agents in North America and Europe [6-9]. In recent reports, a rising trend of KP infection has been observed worldwide [10-12]. In Taiwan, the primary source of infection in endophthalmitis during the past 20 years has been KP liver abscess [13].

There is no age or sex predilection in the incidence of endogenous endophthalmitis. Although it is caused by hematogenous spread, endogenous endophthalmitis tends to develop unilaterally, with the right eye more commonly affected, possibly due to the more proximal and direct arterial route from the heart to the right carotid artery. Several risk factors for endogenous endophthalmitis have been reported, including chronic metabolic diseases, immunosuppression, malignancy, intravenous drug abuse, longterm presence of an intracorporeal foreign body, and invasive surgery [13-16]. According to a worldwide systematic survey of endogenous bacterial endophthalmitis, diabetes was the most common predisposing medical condition, and liver abscess was the most common extraocular focus of infection [17]. A possible association between absence of posterior vitreous detachment and severe endogenous endophthalmitis was reported in a case series [18].

Ocular symptoms of endogenous endophthalmitis, including ocular pain, redness, swelling, and discharge as well as blurred vision and floaters, are non-specific and their occurrence depends on the severity and extent of infection. Panophthalmitis, a severe form of endophthalmitis, can involve tissue adjacent to the orbit, but the infected eye alone does not ever serve as the source of bacteremia or fungemia. The presence of bacteremia or fungemia indicates the possibility of other infective loci in the body. Even in patients with severe endophthalmitis, systemic symptoms are often non-specific and include malaise, nausea, loss of appetite or weight, abdominal discomfort, fever, chills, and rigor. Hence, in patients without obvious systemic infection, diagnosis of the systemic foci can be missed or delayed, and the patients are considered as having ocular disease alone, leaving possibly devastating disease, such as endocarditis and liver abscess, unrecognized. On the other hand, ocular signs and symptoms might be neglected in seriously ill patients. Whether routine ocular screening should be performed in patients with sepsis is controversial due to conflicting results regarding the incidence of endophthalmitis in septic patients [19-21].

The diagnosis of endophthalmitis depends mostly on the clinical findings of ophthalmological examination. In the absence of ocular trauma or surgery, systemic investigations should be performed immediately to look for possible sources of infection or presence of malignancy such as retinoblastoma in children, leukemia, or intraocular lymphoma, which might masquerade as panuveitis. Imaging, including sonography or computed tomography of the abdomen, is helpful in identifying liver abscess. Testing for human immunodeficiency virus is necessary to rule out the possibility of opportunistic infections. The pathogen should be identified using microbiological or molecular techniques for staining and culture of specimens from the eye or bodily fluid (blood, urine, cerebrospinal fluid). Polymerase chain reaction is also helpful in supporting the diagnosis. Since ocular and systemic symptoms of endophthalmitis are typically non-specific, early diagnosis relies on the alertness of ophthalmologists and other physicians. Physicians should be advised to assess the eyes of septic patients, especially in those who are unconscious. Ophthalmologists should be aware that the eye can be the primary site of initial manifestation of major systemic infection. In our previous retrospective report including 602 patients with KP liver abscess, 19\% (8 / 42) of patients with endogenous KP endophthalmitis first visited an ophthalmologist for ocular symptoms [15].

Owing to the rapid progression and poor prognosis of endophthalmitis, treatment should be started empirically without waiting for laboratory confirmation. Treatment should typically be started with systemic broad-spectrum antibiotics combined with intravitreal injection of antibiotics (ceftazidime, $2.25 \mathrm{mg} / 0.1 \mathrm{~mL}$; vancomycin, $1 \mathrm{mg} / 0.1$ $\mathrm{mL}$ ) or anti-fungal agents (amphotericin B, fluconazole, voriconazole). The use of steroids is controversial due to inconclusive results in the literature [22]. Topical cycloplegics, steroids, or hypotensive agents can be used as adjunctive therapy to ameliorate inflammation and pain as well as secondary glaucoma. Pars plana vitrectomy (PPV) is indicated for persistent inflammation, vitreous biopsy, or retinal complication. Owing to advancements in vitreoretinal technology, early vitrectomy is becoming increasingly common for pathogen removal and delivery of medication into the posterior segment, allowing greater preservation 
of useful vision [7,23]. Over the last decade, there have been significant changes in the technology of vitreoretinal surgery. Compared to the traditional 20 -gauge vitrectomy system, microincision vitrectomy surgery causes markedly less intraoperative bleeding and improves procedural efficiency. In addition, advanced illumination and wide-angle viewing systems allow clearer visualization of the fundus through opaque media caused by corneal edema and vitreous opacity.

The prognosis of endophthalmitis is generally poor $[7,9,13,15,16]$. KP infection and poor initial vision are significantly associated with poor visual outcome. Moreover, patients with KP endophthalmitis have worse visual outcome than those with non-KP endophthalmitis. Diabetes is more prevalent in patients with KP than in those with non$\mathrm{KP}$ endogenous bacterial endophthalmitis, and patients with diabetes are more susceptible to developing metastatic infections arising from a KP liver abscess [13,15]. Furthermore, diabetes is known to interfere with the chemotaxis of polymorphonuclear leukocytes and to impair phagocytosis of capsular serotype $\mathrm{K} 1$ or K2 of KP in type 2 diabetic patients with poor glycemic control $[24,25]$. Results from an animal study showed that the diabetic ocular environment facilitates the development of endogenous KP endophthalmitis, possibly via increased permeability of the blood-retinal barrier [26]. In addition, the eye may be overrun with KP long before ocular symptoms or even systemic symptoms of liver abscess are observed. Results of our previous study suggested that the absence of diabetes is an important protective factor against undertreatment of endogenous endophthalmitis secondary to KP liver abscess [27].

In addition to being the most common cause of endogenous endophthalmitis in Asia, KP is also a unique and leading cause of pyogenic liver abscess (PLA) in Taiwan. According to an analysis of nationwide health insurance data, $75.5 \%$ of PLA in Taiwan is KP related PLA, and the rate of endogenous bacterial endophthalmitis development in patients with PLA is 10 -fold higher than the rate in those without ( $0.84 \%$ vs. $0.07 \%)$ [28]. Moreover, the clinical presentation of endogenous KP endophthalmitis varied greatly due to differing patient, pathogenic, and environmental factors (Fig. 1A-1D).

In comparison to endogenous bacterial endophthalmitis, fungal endophthalmitis is much rarer, with Candida and Aspergillus species being the most commonly reported causes [29]. Most cases of Candida endophthalmitis are
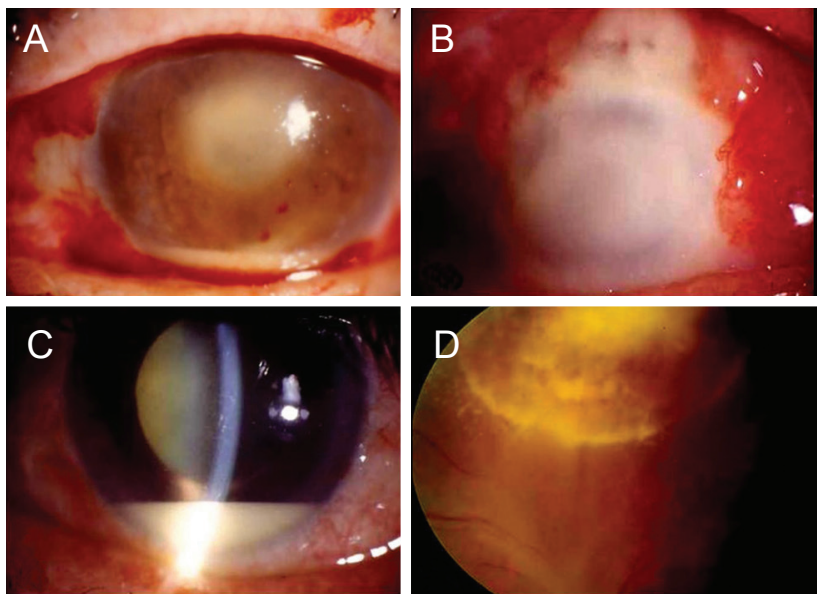

Fig. 1. (A) A 70-year-old female with diabetes developed endogenous endophthalmitis secondary to Klebsiella pneumoniae (KP) liver abscess. Clinical presentation included typical papillary hypopyon. (B) A 75-year-old diabetic male visited an ophthalmologist before the diagnosis of KP liver abscess. Clinical examination revealed severe panophthalmitis without history of ocular trauma or surgery. The eye was lost despite treatment with systemic and intravitreal antibiotics. (C) A 63-year-old male with hypertension and history of endocarditis and liver abscess treated elsewhere 3 months prior. Ocular examination showed hypopyon, lens opacity, and vitreous opacity. Inflammation resolved after combined pars plana vitrectomy, extracapsular cataract extraction, and administration of intravitreal antibiotics. (D) A 45-year-old male with poorly controlled diabetes developed bilateral endophthalmitis secondary to KP liver abscess. Endophthalmitis resolved after systemic antibiotics and one treatment with intravitreal ceftazidime.

endogenous and arise as a complication of candidemia. Risk factors for Candida endophthalmitis, which is most frequently caused by Candida albicans followed by Candida tropicalis, include presence of diabetes or neutropenia; use of an indwelling central catheter, total parenteral nutrition, broad-spectrum antibiotics, or steroids; and recent history of abdominal surgery or abortion. The majority of cases show typical clinical features, such as a creamy white, well-circumscribed lesion involving the retina and choroid in the posterior pole. Multiple satellite lesions can occur, creating a "string of pearls" appearance. Cases of pregnancy-related Candida endophthalmitis have been reported, highlighting its occurrence in healthy immunocompetent women [30-32]. The underlying cause might be increased vaginal fungal colonization during pregnancy due to hormonal changes.

Mold is another possible, though rare, cause of endogenous endophthalmitis. Mold-related endophthalmitis mostly occurs in patients who are severely immunocompromised 

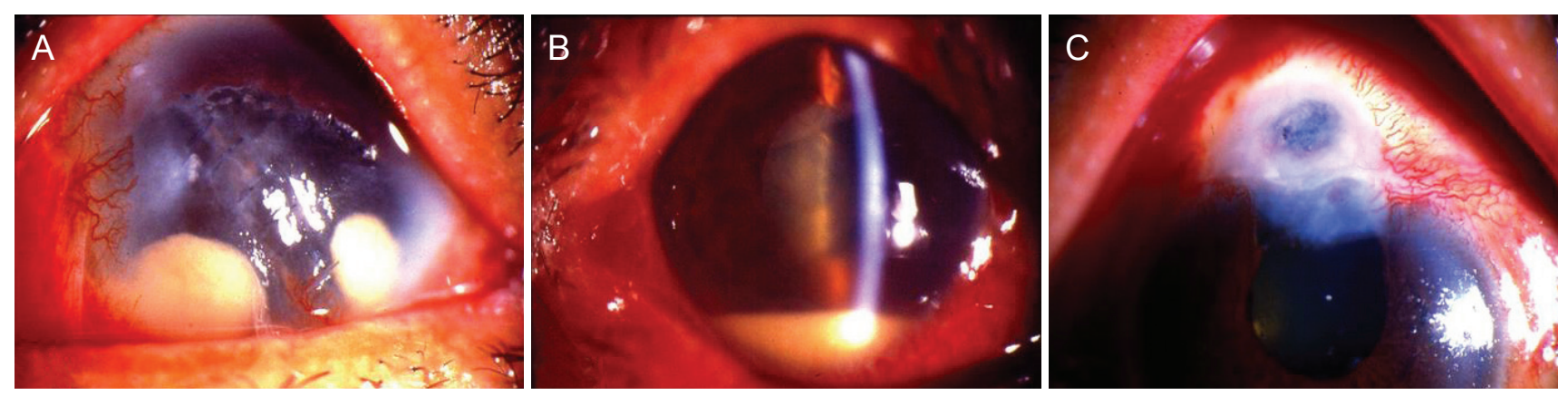

Fig. 2. (A) A case of severe exogenous fungal endophthalmitis secondary to eyeball rupture post repair. (B) A typical case of acute postoperative endophthalmitis after phacoemulsification. (C) A case of chronic bleb-related endophthalmitis.

or are drug abusers, and its diagnosis is frequently delayed due to concurrent critical systemic conditions, especially in cancer patients. Filamentary fungal endophthalmitis has been reported to be a strong risk factor for death in cancer patients [33].

\section{Exogenous Endophthalmitis}

Exogenous endophthalmitis occurs when infecting organisms gain entry into the eye by direct inoculation, such as from intraocular surgery, penetrating trauma, or contiguous spread from adjacent tissues. Postoperative endophthalmitis is a detrimental complication following surgery and is classified as acute or chronic based on the interval between surgery and onset ( $\geq 6$ weeks). Sources of infection in postoperative endophthalmitis include bacteria colonized on the patient's eyelid margin and conjunctiva, healthcare personnel, surgical instruments, solutions, and intraocular lens (Fig. 2A-2C).

The incidence of endophthalmitis after cataract surgery has been reported to range from $0.03 \%$ to $0.2 \%$ [34]. Serial studies from the Bascom Palmer Institute showed a decrease in incidence in the most recent series. The features and outcomes of endophthalmitis associated with clear corneal cataract surgery were similar to those reported in the Endophthalmitis Vitrectomy Study, which are associated with scleral incisions, but time to diagnosis was longer with clear corneal incisions [35].

A systemic review of 148,643 vitrectomy cases reported an incidence of endophthalmitis of $0.046 \%$ [36]. Although endophthalmitis was one of the main initial concerns of sutureless microincision vitrectomy surgery, smaller gauge port sizes were not found to be a risk in recent series. An increase in risk for endophthalmitis has been reported with immunosuppression (odds ratio, 19.0; $p=0.001$ ) and use of preoperative topical steroids (odds ratio, 131.4; $p<0.001$ ) [37].

With the advent of anti-vascular endothelial growth factor agents, intravitreal pharmacotherapy has become increasingly common. Both infectious and non-infectious endophthalmitis have been reported after intravitreal anti-vascular endothelial growth factor treatment. A meta-analysis including 14,886 intravitreal procedures published in 2004 showed that the incidence of endophthalmitis was $0.2 \%$ per injection and $0.5 \%$ per eye [38]. However, a recent large series found the incidence of endophthalmitis per injection of anti-vascular endothelial growth factor agent to be very low and in the range of $0.05 \%$ or less [39]. The updated standard aseptic procedure, which includes use of a surgical mask, $5 \%$ povidone-iodine solution, and an eye speculum, has helped to reduce the risk of infection related to intravitreal injections. Standard sterilization technique in an operating room with laminar air flow was associated with an even lower rate of endophthalmitis of $0.0074 \%$, as reported by a joint survey of three European sites [40]. There is no evidence supporting the benefit of antibiotic prophylaxis in preventing endophthalmitis after intravitreal injection [41].

Many of the current treatment guidelines originate from the Endophthalmitis Vitrectomy Study, a randomized, multicenter clinical trial that treated patients with bacterial endophthalmitis with intravitreal vancomycin and amikacin; subconjunctival vancomycin, ceftazidime, and dexamethasone; or systemic corticosteroids [42]. The study reported a benefit from PPV only in eyes with visual acuity of light perception. Some treatment concepts have changed since the Endophthalmitis Vitrectomy Study was pub- 
lished. Intravitreal amikacin is now avoided due to the risk of aminoglycoside toxicity. Antibiotics with broad-spectrum coverage of gram-positive and gram-negative organisms should be started before the results of culture are confirmed. Intravitreal treatment with ceftazidime and vancomycin is currently preferred. Microincision vitrectomy surgery minimizes manipulation of the conjunctiva and causes less intraoperative bleeding and patient discomfort in an inflamed eye compared to standard 20-gauge vitrectomy. Similar to the management of endogenous endophthalmitis, surgical intervention tends to be performed early, and early vitrectomy is the trend in the management of exogenous endophthalmitis. A procedure that combines limbal-based vitrectomy and PPV can facilitate removal of anterior chamber fibrin and hypopyon, allowing safe and optimal posterior vitrectomy [43]. Removal of the intraocular lens or the entire capsule might be necessary in refractory cases.

The incidence rate of post-traumatic endophthalmitis following open-globe injury has been reported to be $0 \%$ to $16.5 \%$, and risk factors include a retained intraocular foreign body, lens rupture, and delayed repair [44]. The causative microorganisms are derived from either the normal flora surrounding the eyelid area gaining entry after a delay in primary wound closure or are carried into the wound by contaminated injury-causing objects. Prophylactic treatment, including intravitreal antibiotics, systemic broad-spectrum antibiotics, and topical antibiotics, is recommended in high-risk open-globe injuries. Early wound closure and comprehensive antibiotic treatment can improve visual acuity outcome. Concurrent retinal detachment with endophthalmitis can be successfully managed with vitrectomy and intravitreal antibiotics along with a long-acting gas or silicone oil tamponade.

Exogenous endophthalmitis caused by fungus is less common than that caused by bacteria. Exogenous fungal endophthalmitis results from direct inoculation of fungus into the eye, and mostly occurs after eye surgery or trauma or due to extension of fungal infection of the cornea (termed "fungal keratitis" or "keratomycosis"). Although previous reports have suggested that mold was the most common causative agent for exogenous fungal endophthalmitis, a recent report from Korea showed that Candida species were the most common causative organisms [4547]. The discrepancy might be due to differences in the immune status of the patients, pattern of trauma, or spectrum of keratitis. Geographic and environmental factors might also play a role, as molds are more common in tropical regions. Moreover, recent outbreaks of fungal endophthalmitis associated with contaminated compounded brilliant blue $\mathrm{G}$ and triamcinolone have been reported [48]. Management of exogenous fungal endophthalmitis depends on the general condition of the patient and has evolved with improvements in modern vitreoretinal surgical techniques. The combination of PPV and intravitreal antifungal agents has been the most common treatment method for fungal endophthalmitis. Removal of foreign materials, including the intraocular lens, might be necessary in refractory cases. The prognosis of fungal endophthalmitis is usually dismal, but hopefully will improve with the availability of newer generation antifungal agents such as voriconazole.

\section{Conclusions}

Endophthalmitis is a significant eye disease that can cause devastating consequences. The prognosis is usually extremely poor, even with prompt diagnosis and treatment. Thorough evaluation of clinical presentation, systemic symptoms, and history is essential to the diagnosis of endophthalmitis. Most importantly, ophthalmologist awareness of and alertness to the disease are critical. Laboratory examinations are important adjuncts, especially in immunocompromised patients. Early diagnosis and proper treatment of endophthalmitis are keys to saving the eye and possibly the life of the patient.

\section{Conflict of Interest}

No potential conflict of interest relevant to this article was reported.

\section{References}

1. Lemley CA, Han DP. Endophthalmitis: a review of current evaluation and management. Retina 2007;27:662-80.

2. Chee SP, Jap A. Endogenous endophthalmitis. Curr Opin Ophthalmol 2001;12:464-70.

3. Shrader SK, Band JD, Lauter CB, Murphy P. The clinical spectrum of endophthalmitis: incidence, predisposing factors, 
and features influencing outcome. J Infect Dis 1990;162:11520.

4. Krause L, Bechrakis NE, Heimann H, et al. Incidence and outcome of endophthalmitis over a 13 -year period. Can J Ophthalmol 2009;44:88-94.

5. Ramakrishnan R, Bharathi MJ, Shivkumar C, et al. Microbiological profile of culture-proven cases of exogenous and endogenous endophthalmitis: a 10-year retrospective study. Eye (Lond) 2009;23:945-56.

6. Durand ML. Endophthalmitis. Clin Microbiol Infect 2013;19:227-34.

7. Lee S, Um T, Joe SG, et al. Changes in the clinical features and prognostic factors of endogenous endophthalmitis: fifteen years of clinical experience in Korea. Retina 2012;32:977-84.

8. Connell PP, O’Neill EC, Fabinyi D, et al. Endogenous endophthalmitis: 10-year experience at a tertiary referral centre. Eye (Lond) 2011;25:66-72.

9. Wong JS, Chan TK, Lee HM, Chee SP. Endogenous bacterial endophthalmitis: an east Asian experience and a reappraisal of a severe ocular affliction. Ophthalmology 2000;107:1483-91.

10. Karama EM, Willermain F, Janssens X, et al. Endogenous endophthalmitis complicating Klebsiella pneumoniae liver abscess in Europe: case report. Int Ophthalmol 2008;28:111-3.

11. Kashani AH, Eliott D. The emergence of Klebsiella pneumoniae endogenous endophthalmitis in the USA: basic and clinical advances. J Ophthalmic Inflamm Infect 2013;3:28.

12. Odouard C, Ong D, Shah PR, et al. Rising trends of endogenous Klebsiella pneumoniae endophthalmitis in Australia. Clin Exp Ophthalmol 2017;45:135-42.

13. Chen SC, Lee YY, Chen YH, et al. Klebsiella pneumoniae infection leads to a poor visual outcome in endogenous endophthalmitis: a 12-year experience in Southern Taiwan. Ocul Immunol Inflamm 2016 Jul 20:1-8. https://doi.org/10.108 0/09273948.2016.1193616.

14. Park IH, Jun CH, Wi JW, et al. Prevalence of and risk factors for endogenous endophthalmitis in patients with pyogenic liver abscesses. Korean J Intern Med 2015;30:453-9.

15. Sheu SJ, Kung YH, Wu TT, et al. Risk factors for endogenous endophthalmitis secondary to klebsiella pneumoniae liver abscess: 20-year experience in Southern Taiwan. Retina 2011;31:2026-31.

16. Lim HW, Shin JW, Cho HY, et al. Endogenous endophthalmitis in the Korean population: a six-year retrospective study. Retina 2014;34:592-602.

17. Jackson TL, Paraskevopoulos T, Georgalas I. Systematic review of 342 cases of endogenous bacterial endophthalmitis.
Surv Ophthalmol 2014;59:627-35.

18. Umazume K, Suzuki J, Usui Y, et al. Possible relation between lack of posterior vitreous detachment and severe endogenous endophthalmitis. J Ophthalmol 2016;2016:8561379.

19. Dozier CC, Tarantola RM, Jiramongkolchai K, Donahue SP. Fungal eye disease at a tertiary care center: the utility of routine inpatient consultation. Ophthalmology 2011;118:1671-6.

20. Adam MK, Vahedi S, Nichols MM, et al. Inpatient ophthalmology consultation for fungemia: prevalence of ocular involvement and necessity of funduscopic screening. Am J Ophthalmol 2015;160:1078-83.e2.

21. Paulus YM, Cheng S, Karth PA, Leng T. Prospective trial of endogenous fungal endophthalmitis and chorioretinitis rates, clinical course, and outcomes in patients with fungemia. Retina 2016;36:1357-63.

22. Sadiq MA, Hassan M, Agarwal A, et al. Endogenous endophthalmitis: diagnosis, management, and prognosis. $J$ Ophthalmic Inflamm Infect 2015;5:32.

23. Yoon YH, Lee SU, Sohn JH, Lee SE. Result of early vitrectomy for endogenous Klebsiella pneumoniae endophthalmitis. Retina 2003;23:366-70.

24. Mowat A, Baum J. Chemotaxis of polymorphonuclear leukocytes from patients with diabetes mellitus. $N$ Engl J Med 1971;284:621-7.

25. Lin JC, Siu LK, Fung CP, et al. Impaired phagocytosis of capsular serotypes $\mathrm{K} 1$ or K2 Klebsiella pneumoniae in type 2 diabetes mellitus patients with poor glycemic control. $J$ Clin Endocrinol Metab 2006;91:3084-7.

26. Coburn PS, Wiskur BJ, Christy E, Callegan MC. The diabetic ocular environment facilitates the development of endogenous bacterial endophthalmitis. Invest Ophthalmol Vis Sci 2012;53:7426-31.

27. Sheu SJ, Chen YS, Lin HS, et al. A lack of ongoing diabetes is an important factor in preserving eyes from late or suboptimally treated endogenous endophthalmitis secondary to Klebsiella pneumoniae liver abscess. Taiwan J Ophthalmol 2015;5:23-7.

28. Hu CC, Ho JD, Lou HY, et al. A one-year follow-up study on the incidence and risk of endophthalmitis after pyogenic liver abscess. Ophthalmology 2012;119:2358-63.

29. Sridhar J, Flynn HW Jr, Kuriyan AE, et al. Endogenous fungal endophthalmitis: risk factors, clinical features, and treatment outcomes in mold and yeast infections. J Ophthalmic Inflamm Infect 2013;3:60.

30. Tsai CC, Chen SJ, Chung YM, et al. Postpartum endogenous Candida endophthalmitis. J Formos Med Assoc 
2002;101:432-6.

31. Lee JH, Kim JS, Park YH. Diagnosis and treatment of postpartum Candida endophthalmitis. J Obstet Gynaecol Res 2012;38:1220-2.

32. Potasman I, Leibovitz Z, Sharf M. Candida sepsis in pregnancy and the postpartum period. Rev Infect Dis 1991;13:146-9.

33. Lamaris GA, Esmaeli B, Chamilos G, et al. Fungal endophthalmitis in a tertiary care cancer center: a review of 23 cases. Eur J Clin Microbiol Infect Dis 2008;27:343-7.

34. Vaziri K, Schwartz SG, Kishor K, Flynn HW Jr. Endophthalmitis: state of the art. Clin Ophthalmol 2015;9:95-108.

35. Lalwani GA, Flynn HW Jr, Scott IU, et al. Acute-onset endophthalmitis after clear corneal cataract surgery (19962005): clinical features, causative organisms, and visual acuity outcomes. Ophthalmology 2008;115:473-6.

36. Govetto A, Virgili G, Menchini F, et al. A systematic review of endophthalmitis after microincisional versus 20-gauge vitrectomy. Ophthalmology 2013;120:2286-91.

37. Park JC, Ramasamy B, Shaw S, et al. A prospective and nationwide study investigating endophthalmitis following pars plana vitrectomy: clinical presentation, microbiology, management and outcome. Br J Ophthalmol 2014;98:1080-6.

38. Jager RD, Aiello LP, Patel SC, Cunningham ET Jr. Risks of intravitreous injection: a comprehensive review. Retina 2004;24:676-98.

39. Moshfeghi AA, Charalel RA, Hernandez-Boussard T, et al. Declining incidence of neonatal endophthalmitis in the United States. Am J Ophthalmol 2011;151:59-65.e1.
40. Freiberg FJ, Brynskov T, Munk MR, et al. Low endophthalmitis rates after intravitreal anti-vascular endothelial growth factor injections in an operation room: a retrospective multicenter study. Retina 2017 Jan 17. https://doi. org/10.1097/IAE.0000000000001488.

41. Benoist d'Azy C, Pereira B, Naughton G, et al. Antibioprophylaxis in prevention of endophthalmitis in intravitreal injection: a systematic review and meta-analysis. PLoS One 2016;11:e0156431.

42. Microbiologic factors and visual outcome in the endophthalmitis vitrectomy study. Am J Ophthalmol 1996;122:830-46.

43. Xu K, Chin EK, Almeida DR. Five-port combined limbal and pars plana vitrectomy for infectious endophthalmitis. Case Rep Ophthalmol 2016;7:289-91.

44. Ahmed Y, Schimel AM, Pathengay A, et al. Endophthalmitis following open-globe injuries. Eye (Lond) 2012;26:212-7.

45. Wykoff CC, Flynn HW Jr, Miller D, et al. Exogenous fungal endophthalmitis: microbiology and clinical outcomes. Ophthalmology 2008;115:1501-7.

46. Pflugfelder SC, Flynn HW Jr, Zwickey TA, et al. Exogenous fungal endophthalmitis. Ophthalmology 1988;95:19-30.

47. Kim DY, Moon HI, Joe SG, et al. Recent clinical manifestation and prognosis of fungal endophthalmitis: a 7-year experience at a tertiary referral center in Korea. $J$ Korean Med Sci 2015;30:960-4.

48. Mikosz CA, Smith RM, Kim M, et al. Fungal endophthalmitis associated with compounded products. Emerg Infect Dis 2014;20:248-56. 